\title{
Comunicación

\section{Estrategias de interacción y comunicación de showrunners de series de ficción televisiva de Netflix en Twitter ${ }^{1}$ \\ Netflix TV fiction series showrunners' interaction and communication strategies on Twitter}

DOI: https://doi.org/10.32870/cys.v2021.7772

\section{MARÍA-JOSÉ HIGUERAS-RUIZ²}

https://orcid.org/0000-0002-6849-3433JORDI

ALBERICH-PASCUAL 3

https://orcid.org/0000-0001-6871-4614

Este artículo estudia cómo los showrunners se comunican con su audiencia en la red social Twitter. Tras una revisión literaria sobre el panorama televisivo derivado de la implantación de servicios de streaming y redes sociales, se analizan cuantitativa y cualitativamente las técnicas, el contenido y los elementos audiovisuales y textuales que utilizan en Twitter los showrunners de series originales de Netflix. A pesar de la heterogeneidad de su gestión, los resultados reflejan un uso reiterado del hashtag de la serie de televisión, de los tuits informativos/promocionales y de los enlaces externos.

PALABRAS CLAVE: Series de televisión, showrunner, audiencia, Netflix, Twitter.

This paper studies the techniques used by showrunners to communicate with their audience on the social network Twitter. Following a literature review of the television landscape resulting from streaming services and social media, the techniques, content, and audiovisual-textual elements present in the Twitter activity of the showrunners of original Netflix TV series are quantitatively and qualitatively examined. In spite of the heterogeneous activity, the findings indicate repeated use of the TV series' hashtag, informative/promotional twits, and external links.

KEYWORDS: TV series, showrunner, audience, Netflix, Twitter.

Cómo citar este artículo:

Higueras-Ruiz, M. J. \& Alberich-Pascual, J. (2021). Estrategias de interacción y comunicación de showrunners de series de ficción televisiva de Netflix en Twitter. Comunicación y Sociedad, e7772. https://doi.org/10.32870/cys.v2021.7772

1 El presente artículo ha sido elaborado durante la contratación pre-doctoral: "Formación de Profesorado Universitario" (FPU 15/00737) del Ministerio de Educación, Cultura y Deporte del Gobierno de España, y en el marco del Proyecto de Investigación de I+D de Plan Nacional "Transmedialización y crowdsourcing en las narrativas de ficción y no ficción audiovisuales, periodísticas, dramáticas y literarias”, (2018-2020) (Ref. CSO2017-85965-P). Domingo Sánchez-Mesa Martínez y Jordi Alberich-Pascual (2018-2020).

2 Universidad de Granada, España.

mhiguer@ugr.es

3 Universidad de Granada, España.

jalberich@ugr.es

Fecha de recepción: 02/04/20. Aceptación: 27/10/20. Publicado: 17/03/21. 


\section{INTRODUCCIÓN}

Las novedades recientes del modelo de negocio en la industria televisiva han supuesto la revolución del medio, estrechamente relacionada con el avance de Internet durante la última década (Rincón, 2011), la implantación de las plataformas de streaming o VOD (video on demand o video bajo demanda) y de las redes sociales (Lotz, 2016; Van-Esler, 2016).

En el panorama televisivo contemporáneo también destaca la popularidad adquirida por el perfil del showrunner: el productor ejecutivo que lidera el proceso de producción de la serie de televisión, supervisa todas las fases y ostenta la máxima responsabilidad sobre los aspectos ejecutivos y creativos (Higueras-Ruiz et al., 2018). La pertinencia de dicha figura en esta investigación deriva de su labor en los procesos de promoción de las series de ficción, cuando desarrolla funciones para promover una marca personal reconocible por la audiencia que contribuye a la asignación de la autoría de la obra (Mittell, 2015), lo que proporciona un prestigio crítico y cultural a estos profesionales y sus proyectos (Cascajosa-Virino, 2016). Ello motiva la incorporación de los nombres de determinados showrunners en los eslóganes publicitarios como reclamo promocional dentro de las estrategias de marketing desarrolladas por la industria televisiva hollywoodense (Newman \& Levine, 2012).

Por su parte, los servicios de streaming repercuten tanto en la transformación del proceso de creación, producción y distribución de series de ficción (Cascajosa-Virino, 2018), como de las formas y hábitos de consumo televisivo (Guerrero et al., 2017; Lacalle-Zalduendo \& Gómez-Morales, 2017). El contenido se distribuye a través de Internet sin un horario de programación lineal preestablecido, y la audiencia lo ve cuando y desde el dispositivo que prefiera mediante el pago de una cuota (Ojer \& Capapé, 2013).

En este contexto disruptivo apreciamos la significación contemporánea alcanzada por la plataforma Netflix, motivada "por el liderazgo que ejerce en su mercado doméstico, Estados Unidos, así como por su expansión internacional" (Izquierdo-Castillo, 2015, p. 819), y más 
específicamente por el sello Netflix Original:4 contenido original producido o coproducido por el propio operador. Estos proyectos construyen una imagen de marca, fomentan la subscripción al mantenerse en el catálogo de manera exclusiva e indefinida, y dan respuesta a los intereses y gustos de la audiencia al crearse en retroalimentación de los datos extraídos de su propio comportamiento en la plataforma (Cornelio-Marí, 2020; Fernández-Manzano et al., 2016).

En oposición al modelo de negocio diacrónico tradicional, el contenido original de Netflix conlleva la creación, producción y oferta sincrónica de todos los episodios de una temporada. El showrunner es contratado para producir un proyecto televisivo completo y sin un episodio piloto previo: modalidad conocida como straight-to-series (Carrillo-Bernal, 2018). El estreno de la temporada completa elimina la emisión seriada y las esperas semanales de la televisión tradicional, favoreciendo "la fidelización de la audiencia y... una estabilidad en los ingresos publicitarios o de suscripción" (Izquierdo-Castillo, 2015, p. 822), así como que el espectador vea varios capítulos seguidos, práctica denominada atracón televisivo o binge-watching (Matrix, 2014).

La hibridación creciente de Internet y televisión en el marco de la cultura participativa contemporánea también comportará el empoderamiento y significación alcanzada en las redes sociales por las comunidades de seguidores de series de ficción (Pearson, 2010; Scolari \& Establés, 2017). Los espectadores adquieren un renovado y emergente papel activo, configurando un nuevo tipo de audiencia, implicándose en el seguimiento y supervisión colectiva de los proyectos televisivos y reclamando a los agentes de la industria de streaming el uso de nuevas técnicas y estrategias de atracción y estimulación a través de las redes sociales (Phalen, 2018; Wayne, 2018).

4 No todos los proyectos con este distintivo son producidos o coproducidos por Netflix (Wayne, 2018). En este trabajo se seleccionan aquellos que presentan esta característica y son estrenados por primera vez y en temporadas completas en la plataforma. 


\section{TWITTER Y LA INDUSTRIA TELEVISIVA}

DE FICCIÓN CONTEMPORÁNEA

De acuerdo con el estudio anual sobre el uso de Internet a nivel mundial Global Digital 2019 Report, el número de personas en la red asciende a 4388 millones, un 9.1\% más que el año anterior (Kemp, 2019). El citado informe presenta la cifra de 3484 millones de usuarios activos en redes sociales a nivel global, indicando un aumento del $9 \%$ entre enero de 2018 y enero de 2019. El 98\% de ellos visitó una red social o servicio de mensajería, y el $83 \%$ participó activamente en una o más redes sociales, entre las que destacan por su volumen en términos de millones de cuentas activas mensuales: Facebook (2 271), YouTube (1 900), WhatsApp (1 500), Instagram (1 000), Twitter (326), LinkedIn (303) y Snapchat (287).

Ante dichos datos, la industria televisiva ha propiciado la inclusión plena de las redes sociales en las campañas de marketing de las series de televisión, tanto para captar y fidelizar al público, como para gestionar y proteger la marca del producto (McNutt, 2018; Simons, 2014). En esta línea, Segado et al. (2015) expresan:

Según los estudios científicos, las redes sociales no solo han cambiado los patrones de consumo televisivo, sino que también han influido sobre la forma en la que los canales de televisión promueven sus contenidos a través de estas nuevas herramientas (p. 232).

La red social Twitter destaca por su vinculación con el medio televisivo y por la atención recibida por los estudios de audiencias y televisión social (Deller, 2011). La actividad social en torno a un producto audiovisual incrementa su popularidad, influyendo positivamente en su consumo (Halpern et al., 2016) y en sus posibilidades de renovación (Phalen, 2018). La capacidad de Twitter para promocionar proyectos audiovisuales y atraer y mantener espectadores, reforzando su implicación, resulta especialmente interesante a las plataformas VOD. En la sociedad red, "para los contenidos emitidos por streaming, ser visto es menos significativo que el hecho de que se hable acerca de ellos, de forma que motive a las personas a subscribirse y mantenerse en este servicio" (Navar-Gill, 2017, p. 425). 
El interés para los responsables de cadenas, productoras y programas en los comentarios, las respuestas y las menciones de Twitter relacionadas con el mundo de la televisión (Gallego, 2013) promueve que estos apliquen estrategias de enganche, como la emisión de tuits variados, la incorporación de enlaces externos o el uso de elementos audiovisuales (Quintas-Froufe \& González-Neira, 2014). En el caso del perfil de Netflix España, las características de los tuits con mayor engagement son la creación de contenido original, el uso de lenguaje propio y la atención al humor y al suspense (Fernández-Gómez \& Martín-Quevedo, 2018).

Twitter resulta notablemente útil en relación con las series de ficción porque ayuda a mantener e incrementar el deseo de continuar hablando de ellas entre episodios y temporadas. Las cadenas de televisión y plataformas de distribución crean perfiles donde promueven iniciativas para implicar a la audiencia (Latorre-Martínez et al., 2018), así como cuentas específicas para cada serie de televisión asociadas a los miembros de su writers' room (Navar-Gill, 2017). Dichos perfiles suelen estar gestionados por profesionales denominados community managers (Castro-Mariño, 2016), que utilizan el hashtag correspondiente para promocionar el proyecto televisivo.

Conviene destacar igualmente la pertinencia y posibilidades del uso de Twitter de forma paralela a la emisión del programa (Guo, 2019). Las principales funciones en este caso son la comunicación con otros espectadores y la valoración del contenido y sus actores (Buschow et al., 2014). De acuerdo con Halpern et al. (2016), mientras la audiencia prefiere acudir a Facebook para informarse sobre los programas que se consumen, Twitter es la opción elegida para conocer las opiniones del resto de los usuarios.

Los productos que se emiten en televisión y la información que fluye por las redes sociales se retroalimentan (García-Avilés, 2012). Los comentarios de los espectadores suponen una retroalimentación rápida y directa para que los showrunners realicen los cambios oportunos en el proyecto (Harrington et al., 2013; James, 2017), y los retuits resultan altamente pertinentes para la difusión y credibilidad de los contenidos televisivos (Lin \& Peña, 2013). 
Los perfiles personales de los showrunners son habitualmente accesibles a través de Twitter (Marwick \& boyd, 2010). Su presencia, reconocimiento y popularidad en esta red social les convierte en ocasiones en decisivos para la propia imagen pública de la serie de ficción, pudiendo llegar a ser considerados celebridades (Phalen, 2018). Se favorece con ello que estos profesionales utilicen sus cuentas como espacios para construir una marca de autor que muestran a sus seguidores a través del diseño del perfil en la red social y mediante la aplicación de determinadas técnicas, contenidos y elementos en los mensajes que emiten, para desarrollar estratégicamente sus conexiones con la audiencia de los proyectos que producen (Higueras-Ruiz et al., 2019; Navar-Gill, 2018).

\section{OBJETIVOS Y METODOLOGÍA}

El objetivo principal de la presente investigación es determinar las estrategias que los showrunners de series de ficción estadounidenses producidas por Netflix emplean en la interacción y comunicación con los espectadores a través de Twitter. Con esta finalidad hemos procedido a revisar cuantitativa y cualitativamente qué: 1) técnicas; 2) tipo de contenido; y 3) elementos audiovisuales y textuales incluyen los tuits y retuits emitidos por los showrunners para conectar con su audiencia, en relación tanto con la cuenta de la serie de ficción como con la plataforma de Netflix.

Se ha establecido como muestra de estudio los perfiles oficiales -con la insignia de cuenta verificada- en la red social Twitter de los showrunners de series estadounidenses de ficción televisiva de imagen real producidas -o coproducidas- por la plataforma de streaming Netflix, que forman parte de su catálogo e incluyen la etiqueta $A$ Netflix Original Series. La muestra final se ha limitado por razones de actualidad a los showrunners de series cuyas primeras o sucesivas temporadas han sido estrenadas entre el 1 de junio de 2018 y el 30 de junio de 2019.

Puesto que la implicación e interacción con la audiencia a través de las redes sociales es especialmente pertinente durante el periodo de promoción anterior a la emisión del proyecto televisivo (Gallego, 2013), se ha ajustado el trabajo de campo y el tiempo de monitorización específico para la cuantificación de los tuits y retuits de cada perfil, y el 
examen de los mismos, a las cuatro semanas previas al estreno en la plataforma de la temporada completa de la serie.

La selección del mercado estadounidense está motivada por el mayor reconocimiento de la propia figura del showrunner en dicha industria, pionera en la adopción de las nuevas plataformas (Ojer \& Capapé, 2013); por las variantes introducidas en la producción de series de ficción, derivadas de la popularidad y las particularidades del servicio de Netflix y de su contenido original (Jenner, 2014); así como por la notable conexión entre la televisión norteamericana y la red social Twitter (Segado et al., 2015).

Finalmente, tomando en consideración las aportaciones previas de Lin y Peña (2013), Buschow et al. (2014), Quintas-Froufe y González-Neira (2014, 2016), Fernández-Gómez y Martín-Quevedo (2018) y Navar-Gill (2017), se exponen los resultados obtenidos del análisis cuantitativo y cualitativo de los perfiles de Twitter y de los tuits monitorizados, a través de un conjunto de tablas de elaboración propia confeccionadas en relación con los objetivos y elementos de revisión propuestos.

Tras aplicar los criterios de selección previstos: 1. Título de la serie de ficción de Netflix; 2. Temporada de estreno; 3. Fecha de estreno; 4. Showrunner/s; y 5. Twitter oficial, la muestra de estudio de perfiles de showrunners en Twitter se compone de 25 cuentas verificadas, aunque examinamos 26 resultados debido al estreno de dos temporadas de la misma serie durante el tiempo delimitado.

En primer lugar, se lleva a cabo una aproximación descriptiva de los datos numéricos y visuales de los perfiles analizados. A continuación, se busca dar respuesta a las tres preguntas de investigación, mediante el conteo manual y análisis textual de los tuits emitidos:

P1. ¿Qué técnicas incluyen los tuits y retuits emitidos por los showrunners de la muestra en relación con la serie de ficción y con la plataforma de Netflix? Se contabilizan los tuits que incluyen el hashtag, menciones o retuits a la cuenta oficial de la serie de televisión producida por cada showrunner, y a la cuenta oficial de Netflix en Estados Unidos.

P2. ¿Qué tipología de contenido presentan los tuits emitidos por los showrunners de la muestra? Se analizan los mensajes emitidos, cla- 
sificándolos según seis categorías de contenido seleccionadas de acuerdo con la revisión teórica: información/promoción, participación, llamamiento/ayuda, pregunta, agradecimiento u otros temas.

P3. ¿Qué elementos audiovisuales y textuales presentan los tuits emitidos por los showrunners de la muestra? Se atiende a la inclusión de hashtags, emoticonos, fotografías, videos, gifs y enlaces empleados en los tuits.

Los resultados obtenidos dan respuesta a estas cuestiones en relación con la base teórica anteriormente expuesta. Asimismo, permiten determinar las posibilidades de la actuación en Twitter de los creadores de series de ficción para la construcción de una imagen de marca que contribuya a su consideración autoral y al reconocimiento crítico y cultural por parte de la audiencia.

\section{ANÁLISIS Y RESULTADOS}

Para la descripción de los perfiles de la muestra observamos los datos numéricos: 1. Fecha de creación del perfil; 2. Número de tuits (Tw); 3. Número de seguidos ( $\mathrm{Si}$ ); 4. Número de seguidores (Se); y 5. Número de Me Gusta (Mg); así como los datos descriptivos sobre los medios utilizados para vincularse con la serie de ficción: 1. Imágenes de la serie en la fotografía de perfil (Per); 2. Imágenes de la serie en la fotografía de portada (Por); y 3. Referencias a la posición de showrunner en la biografía (Bib).

La observación de los perfiles revela una notable variedad en su actividad, como muestra el contrastado número de seguidores -desde 2.19 millones a 2436 - y de tuits emitidos -desde 40.9 mil a 14-. La relación de la interfaz de la cuenta con la serie de televisión también difiere. Destacamos la aparición de dicha referencia en la biografía en el $68 \%$ de los casos y, en menor medida (36\%), en la fotografía de portada. Por el contrario, la mayoría de los showrunners (88\%) no muestran ninguna conexión con la ficción televisiva a través de su fotografía de perfil.

Atendiendo a la fecha de creación de las cuentas, advertimos que en ningún caso coincide con el periodo de promoción previo al estreno del 


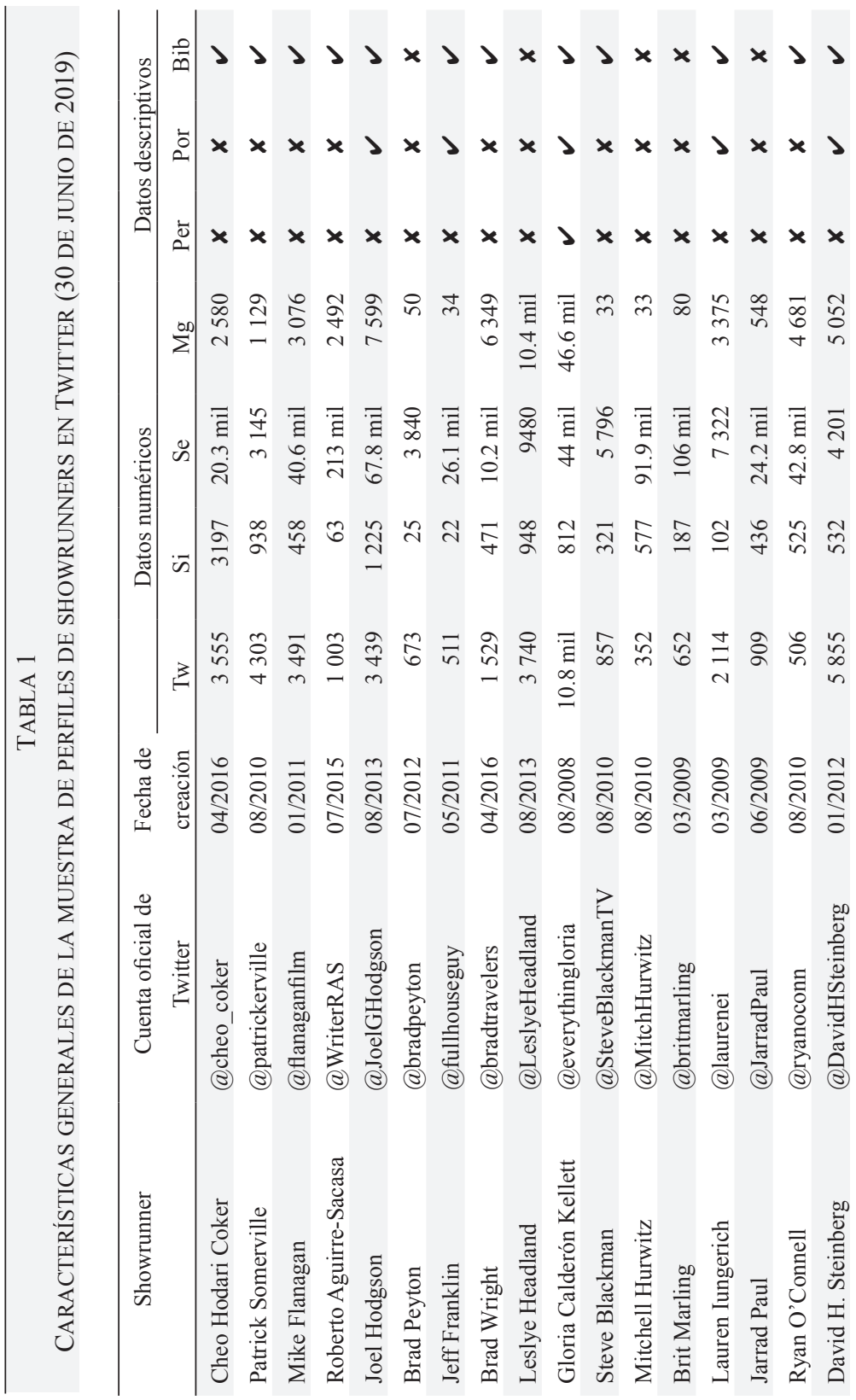




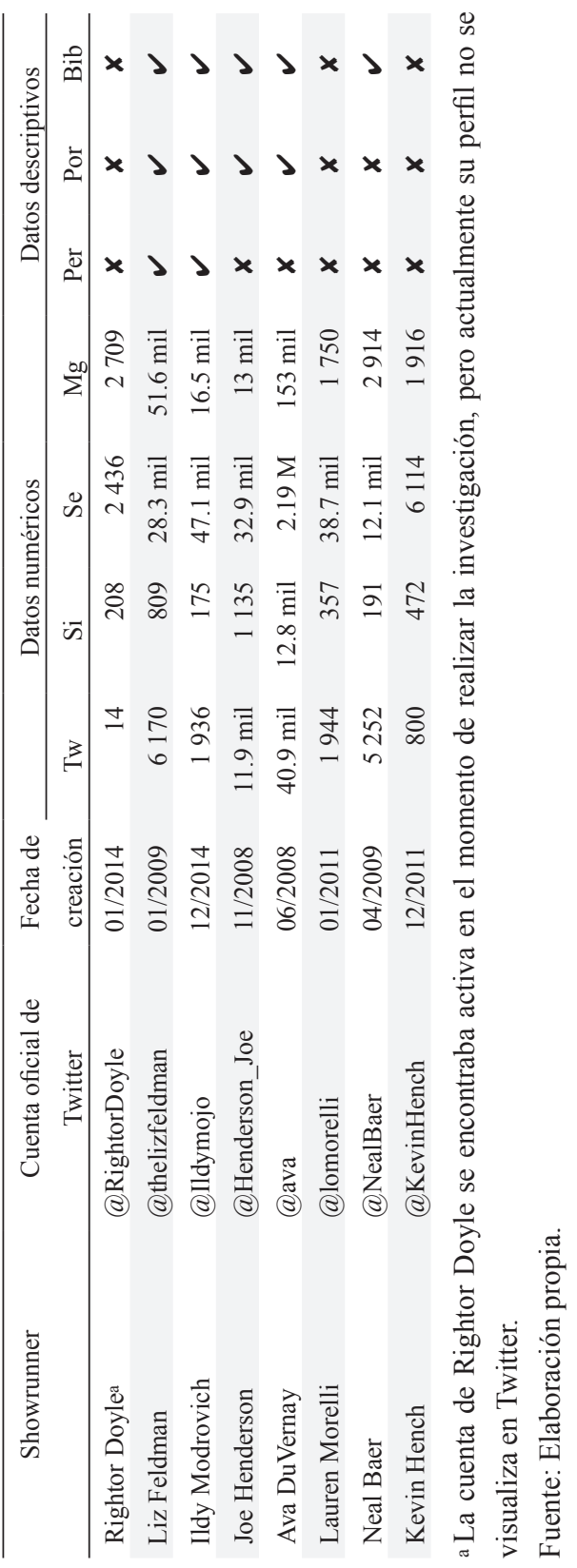


proyecto audiovisual, sino que se sitúa entre dos y once años antes, lo que contribuye a su carácter personal.

\section{TÉCNICAS QUE INCLUYEN LOS TUITS Y RETUITS EMITIDOS POR LOS SHOWRUNNERS}

Cuantificamos los tuits (Tw) y retuits (Rt) emitidos durante la muestra temporal delimitada y los clasificamos según las técnicas seleccionadas: respecto al producto televisivo: 1. Porcentaje de tuits que utilizan el hashtag oficial de la serie de televisión (Hsg); 2. Porcentaje de tuits que mencionan la cuenta oficial de la serie de televisión (Men); 3. Porcentaje de retuits de la cuenta oficial de la serie de televisión (Rt); y a la plataforma de Netflix en Estados Unidos: 4. Porcentaje de tuits que utilizan el hashtag oficial de la plataforma de Netflix Estados Unidos (Hsg); 5. Porcentaje de tuits que mencionan la cuenta oficial de Netflix Estados Unidos (Men), y 6. Porcentaje de retuits de la cuenta oficial de Netflix Estados Unidos (Rt).

Los resultados evidencian una actividad variada en la emisión de tuits y retuits, y en la relación con la cuenta de la serie de televisión y de la plataforma de Netflix de Estados Unidos. A pesar de que el 50\% de los perfiles alcanzan la suma de 28 tuits y retuits en total -mostrando una media de un tuit o retuit al día-, hallamos showrunners cuyo uso de la red social es destacadamente escaso.

Más de la mitad de las cuentas (61.53\%) incluyen el hashtag de la serie de televisión en al menos una ocasión, pero únicamente dos perfiles utilizan dicha etiqueta en más de la mitad de sus tuits -@Ildymojo (81.21\%) y@SteveBlackmanTV (63.15\%)-.

Hallamos cifras inferiores si atendemos a las menciones a la cuenta de la serie de televisión: el 50\% de los perfiles muestran la mención como mínimo una vez, pero ninguno lo hace en la mitad de sus tuits. Los retuits también son empleados al menos una vez por el 50\% de los showrunners, pero de forma notablemente limitada.

Los datos revelan poca interacción con la cuenta de Netflix Estados Unidos: como mínimo en una ocasión el $23.07 \%$ de los perfiles utilizan el hashtag, el $45.30 \%$ la mencionan y el $3.84 \%$ comparten sus contenidos. 


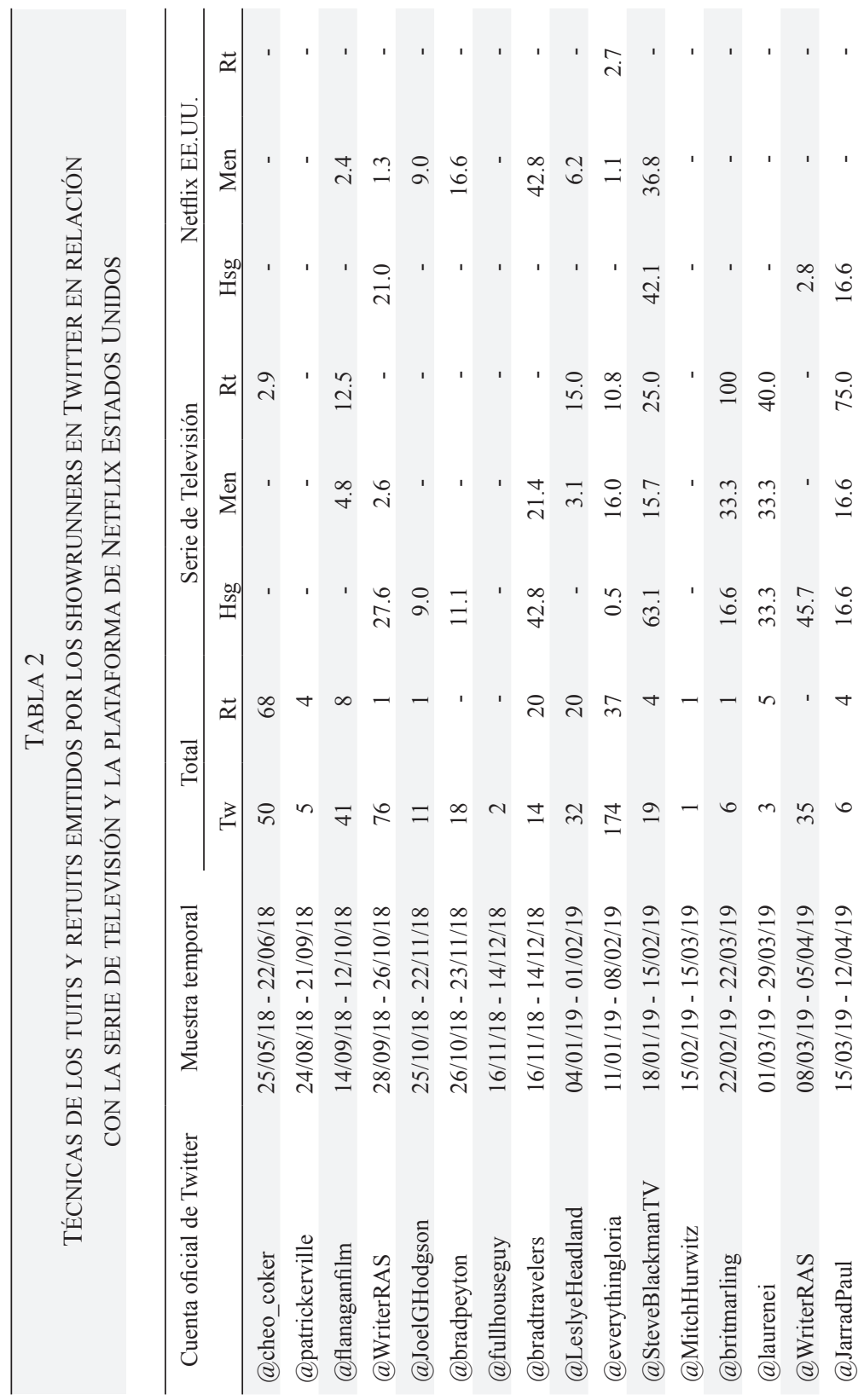




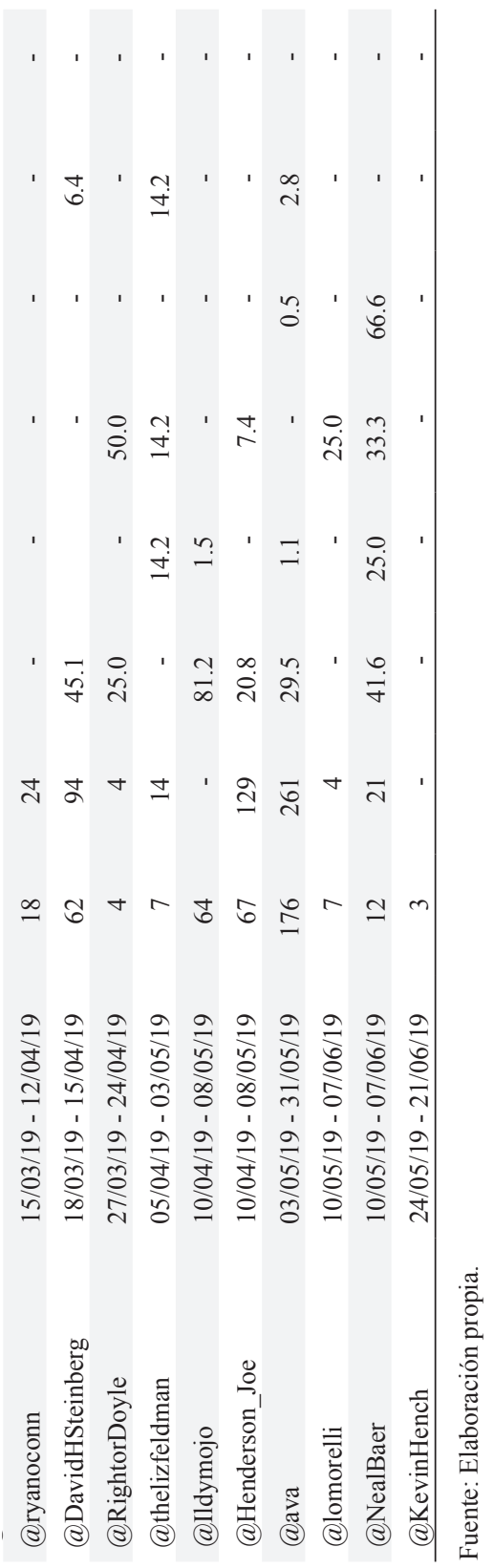




\section{TIPOS DE CONTENIDO QUE INCLUYEN}

\section{LOS TUITS EMITIDOS POR LOS SHOWRUNNERS}

Se presenta el porcentaje de tuits emitidos durante la muestra temporal, clasificados en las siguientes categorías de contenido no excluyentes entre sí: 1. Información/promoción; 2. Participación; 3. Llamamiento/ ayuda; 4. Pregunta; 5. Agradecimiento; y 6. Otros temas.

Los resultados revelan que el tipo de contenido que caracteriza los tuits emitidos por los showrunners es muy similar entre las cuentas. En más de la mitad de los perfiles (57.69\%) al menos la mitad de los tuits responden a la categoría información/promoción sobre la serie de televisión: enlaces al tráiler, cuenta atrás de la fecha de estreno, fotografías del proceso de producción, y críticas y noticias de otros medios. Destacamos el modelo de tuit del día previo al estreno del proyecto:

Una foto más para celebrar el lanzamiento de \#CAOS que va a suceder a medianoche en \#Netflix. ¡Kiernan y Chance, en el primer día de rodaje! ¡iEra la escena de la araña!! Ahora todos ellos han crecido, pero entonces, ¡parecían bebés! ¡Los quiero mucho! \#Sabrina (Aguirre-Sacasa, 2018).

Los tuits acerca de otros temas también son notables: el $46.15 \%$ de las cuentas emplean dicho contenido como mínimo en el 50\% de sus tuits. Observamos el apoyo a otros proyectos audiovisuales, las noticias sobre deporte o política, y las opiniones sociales, ideológicas o culturales que, en algunos casos, conectan con la serie de televisión:

Estados Unidos nunca fue genial. Siempre había alguien en la zona baja. Alguien siendo oprimido, esclavizado, internado, enjaulado, deportado. ¿Cómo sobrevivieron esas personas? Lazos familiares. Tanto familia genética, como comunidad. De eso trata \#QUEENSUGAR. Nuestra familia regresa el 12 de junio (DuVernay, 2019).

Los tuits de agradecimiento son empleados al menos una vez por el $57.69 \%$ de los perfiles. Estos mensajes pueden ir dirigidos tanto al público como a todos los profesionales involucrados en la producción de la serie: 
TABLA 3

TIPOS DE CONTENIDO DE LOS TUITS EMITIDOS POR LOS SHOWRUNNERS EN TWITTER

\begin{tabular}{|c|c|c|c|c|c|c|}
\hline \multirow{2}{*}{$\begin{array}{c}\text { Cuenta oficial de } \\
\text { Twitter }\end{array}$} & \multicolumn{6}{|c|}{ Categorías de contenido } \\
\hline & 1 & 2 & 3 & 4 & 5 & 6 \\
\hline @cheo_coker & 54.0 & - & - & - & 2.0 & 46.0 \\
\hline @patrickerville & - & - & - & - & - & 100 \\
\hline @flanaganfilm & 82.9 & 2.4 & - & - & - & 17.0 \\
\hline @WriterRAS & 30.2 & 1.3 & - & - & - & 68.4 \\
\hline @JoelGHodgson & 63.6 & 27.2 & - & - & 18.1 & 18.1 \\
\hline @bradpeyton & 33.3 & - & - & - & 5.5 & 55.5 \\
\hline @fullhouseguy & 50.0 & - & - & - & - & 50.0 \\
\hline (a)bradtravelers & 50.0 & - & 14.2 & 7.1 & 14.2 & 28.5 \\
\hline @LeslyeHeadland & 3.1 & 3.1 & 6.2 & 6.1 & 3.1 & 78.1 \\
\hline @everythingloria & 18.3 & 4.5 & 1.1 & 4.5 & 8.6 & 67.2 \\
\hline @SteveBlackmanTV & 73.6 & 10.5 & - & - & 10.5 & 21.0 \\
\hline @MitchHurwitz & - & - & - & - & - & 100 \\
\hline @britmarling & 66.6 & - & - & - & - & 33.3 \\
\hline (a)laurenei & 66.6 & - & - & - & - & 33.3 \\
\hline @WriterRAS & 40.0 & 2.8 & - & 11.4 & - & 54.2 \\
\hline @JarradPaul & 66.6 & - & - & - & - & 33.3 \\
\hline @ryanoconn & 33.3 & 16.6 & - & 5.5 & 5.5 & 61.1 \\
\hline @DavidHSteinberg & 58.0 & 4.8 & 1.6 & - & 12.9 & 35.4 \\
\hline @RightorDoyle & 50.0 & - & - & - & 50.0 & - \\
\hline @ thelizfeldman & 57.1 & - & - & - & 28.5 & 42.8 \\
\hline @Ildymojo & 67.1 & 1.56 & 3.1 & 7.8 & 10.9 & 17.1 \\
\hline @Henderson_Joe & 22.3 & 2.9 & - & 1.4 & 1.4 & 76.1 \\
\hline @ava & 32.9 & - & 0.5 & 1.1 & 10.2 & 57.3 \\
\hline @1omorelli & 28.5 & - & - & - & 14.2 & 57.1 \\
\hline @NealBaer & 66.6 & 41.6 & - & 8.3 & - & 25.0 \\
\hline$@$ KevinHench & 66.6 & - & - & - & - & 33.3 \\
\hline
\end{tabular}

Fuente: Elaboración propia. 
Hace un año fuimos cancelados. ¡iHoy \#LuciferTemporada4 está en Netflix!! Estos episodios son para ti, Lucifans. Gracias por luchar tan duro para que pudiéramos hacerlo. \#Lucifer (Henderson, 2019).

Hallamos datos escasos respecto a las categorías de contenido más relacionadas con la audiencia -participación, llamamiento/ayuda, pregunta- que en ninguna cuenta superan el $50 \%$ de sus tuits. No obstante, encontramos ejemplos significativos de mensajes que incitan a la participación en eventos y actividades promocionales:

Cerca de 5808 Sunset Blvd. @OneDayAtATime ¡Camión de comida gratis!! ¡iVen a comer y chocamos los cinco!! (Calderón Kellett, 2019).

Tuits que piden a los espectadores ayuda para la difusión de la serie de televisión:

¡Lanzamiento de \#Travelers este viernes 14 de diciembre! Así que, si te gusta la temporada 3, ¡díselo al mundo! Buenas críticas y calificaciones en IMDb y Rotten Tomatoes pueden ayudarnos mucho. (Y si no te gusta, bueno, por favor, no se lo digas a nadie;)@TRVLRSseries@netflix@ EricMcCormack@Carrie_Mudd (Wright, 2018).

Tuits que incorporan preguntas para estimular a la audiencia:

¿Estás viendo doblesdobles? Entonces debes estar viendo el episodio 119 de \#Sabrinanetflix. ¿¿Se cumplirá la profecía?? \#CAOS (Aguirre-Sacasa, 2019).

\section{ELEMENTOS AUDIOVISUALES Y TEXTUALES}

QUE INCLUYEN LOS TUITS EMITIDOS POR LOS SHOWRUNNERS

Cuantificamos el porcentaje de tuits emitidos durante la muestra temporal que utilizan los siguientes elementos audiovisuales y textuales no excluyentes entre sí: 1. Hashtags, 2. Emoticonos, 3. Fotografías, 4. Videos, 5. Gifs, y 6. Enlaces. 
Los datos obtenidos muestran una notable preferencia por el uso de enlaces externos: el $30.76 \%$ de las cuentas los incluyen en la totalidad de los tuits emitidos, y el $92.30 \%$ al menos en la mitad. Los enlaces conectan los tuits con páginas web de medios de comunicación audiovisual, o redes sociales de los propios showrunners.

Apreciamos el uso de etiquetas diversas, destacando la de la serie de televisión o diferentes versiones de la misma en función de la temporada promocionada. El 26.92\% de las cuentas utilizan hashtags como mínimo en la mitad de sus tuits, y el 73.07\% lo hacen al menos en una ocasión.

Señalamos un uso menor de emoticonos: el $46.15 \%$ de los perfiles los emplean una o más veces y suelen estar relacionados con la historia de la ficción. Algunos showrunners también publican fotografías: carteles promocionales del proyecto e imágenes del proceso de producción y de los eventos relacionados. El 46.15\% de las cuentas las usan al menos en un tuit.

Por el contrario, los videos y gifs no resultan significativos: únicamente el $19.23 \%$ de los showrunners de la muestra y el $15.38 \%$, respectivamente, incorporan los citados elementos en una ocasión como mínimo.

\section{DISCUSIÓN Y CONCLUSIONES}

En primer lugar, se advierte que la escasa interacción en Twitter de algunos showrunners de la muestra nos impide generalizar las posibilidades comunicativas y promocionales de esta herramienta señaladas en la revisión teórica (Segado et al., 2015). A pesar de que nuestra selección resulta representativa de la actuación en Twitter de showrunners de series originales de Netflix, se revela limitada para realizar afirmaciones más amplias al respecto.

Hallamos datos heterogéneos que abarcan desde cuentas con poca información hasta otras notablemente más activas, que aprovechan los beneficios derivados de las novedades del panorama televisivo para interactuar con la audiencia, promocionar la serie de ficción, y crear y fortalecer su imagen de marca autoral más allá de las aspiraciones comerciales. La red social Twitter proporciona un conjunto de estrategias que favorecen que la audiencia identifique a la figura del showrunner. A 
través de la interfaz de sus perfiles, estos profesionales presentan su conexión con la serie de ficción, especialmente en la biografía, de manera que los seguidores comprenden el liderazgo del showrunner en la producción televisiva y se refuerza su consideración como autor de la obra.

En los resultados referentes a las técnicas, contenidos y elementos utilizados, la ausencia de un patrón visible en la emisión de tuits, el carácter personal de algunos contenidos y la limitada actividad de ciertos perfiles incitan a deducir que no son gestionados por un community manager, lo que contribuye a la atención a la red social como herramienta para configurar la imagen personal de los showrunners.

A pesar de la citada variedad en la estrategia y actuación, los datos obtenidos permiten concluir una preferencia de los showrunners por el uso del hashtag, especialmente de la serie de televisión; los tuits de contenido informativo y promocional, acentuándose la semana previa al estreno del proyecto; y la inclusión de enlaces externos que amplían la información del mensaje.

Estos resultados se asemejan a la estrategia de engagement en Twitter llevada a cabo por el perfil de Netflix España (Fernández-Gómez \& Martín-Quevedo, 2018), y por los perfiles de los programas españoles de televisión Código Emprende, La Voz y Top Chef (Quintas-Froufe \& González-Neira, 2014). Los datos extraídos de ambos estudios coinciden en los beneficios de los tuits variados y el uso de elementos audiovisuales, que asimismo se aprecian en esta investigación.

Sin embargo, en las conclusiones de Navar-Gill (2017) sobre la actividad de las cuentas de Twitter de las salas de guionistas de Jane the Virgin, Faking It y Orange Is the New Black, se destaca una mayor interacción con la audiencia que en los resultados de nuestro análisis. Podemos hallar la razón de este desajuste en el citado trabajo, donde la autora anota que el uso de la cuenta de la sala de guionistas de la serie es más pertinente que el del perfil personal del showrunner, para no comprometer la autoridad de la citada figura en las relaciones del medio con la audiencia (Navar-Gill, 2017, p. 418).

Por otra parte, respecto a la configuración de una marca personal a través de la actividad de los showrunners en la red social Twitter, advertimos la coherencia de la misma con la imagen de la propia cadena o plataforma y del proyecto de televisión. En este caso, se aprecia una 


\section{TABLA 4}

ELEMENTOS AUDIOVISUALES Y TEXTUALES DE LOS TUITS EMITIDOS POR LOS SHOWRUNNERS EN TWITTER

\begin{tabular}{|c|c|c|c|c|c|c|}
\hline \multirow{2}{*}{ Cuenta oficial de Twitter } & \multicolumn{6}{|c|}{ Elementos Audiovisuales y Textuales } \\
\hline & 1 & 2 & 3 & 4 & 5 & 6 \\
\hline @echeo_coker & 2.0 & - & - & - & - & 68.0 \\
\hline$@$ patrickerville & - & - & 20.0 & - & - & 80.0 \\
\hline @)flanaganfilm & - & - & 2.4 & - & - & 92.6 \\
\hline @WriterRAS & 88.1 & 93.4 & 78.9 & 3.9 & - & 3.9 \\
\hline @.JoelGHodgson & 9.0 & - & 36.3 & - & - & 54.5 \\
\hline @bradpeyton & 61.1 & 33.3 & - & - & - & 100 \\
\hline @fullhouseguy & - & - & - & - & 100 & 100 \\
\hline @ bradtravelers & 7.1 & - & - & - & - & 42.8 \\
\hline @LeslyeHeadland & 3.1 & 18.7 & 12.5 & 3.1 & 3.1 & 37.5 \\
\hline @everythingloria & 7.4 & 22.9 & 11.4 & - & - & 36.2 \\
\hline @SteveBlackmanTV & 15.7 & - & - & - & - & 100 \\
\hline$@$ MitchHurwitz & - & - & - & - & - & 100 \\
\hline @britmarling & 16.6 & 16.6 & - & - & - & 100 \\
\hline @1aurenei & 33.3 & - & - & - & - & 33.3 \\
\hline (a)WriterRAS & 100 & 85.7 & 88.5 & 5.7 & - & 2.8 \\
\hline @JarradPaul & 16.6 & - & 16.6 & - & - & 50.0 \\
\hline @ryanoconn & - & - & - & - & - & 61.1 \\
\hline @DavidHSteinberg & 50.0 & 3.2 & 12.9 & 1.6 & 1.6 & 53.2 \\
\hline @ RightorDoyle & 50.0 & 50.0 & - & - & - & 100 \\
\hline @ thelizfeldman & 14.2 & - & - & - & - & 100 \\
\hline @Ildymojo & 87.5 & 45.3 & 28.1 & - & - & 67.1 \\
\hline @Henderson_Joe & 31.3 & 7.4 & 10.4 & - & - & 67.1 \\
\hline @ava & 38.0 & 19.8 & 17.0 & 3.4 & 3.9 & 69.8 \\
\hline @lomorelli & - & 42.8 & - & - & - & 85.7 \\
\hline$@$ NealBaer & 75.0 & - & - & - & - & 100 \\
\hline @KevinHench & - & - & - & - & - & 66.6 \\
\hline
\end{tabular}

Fuente: Elaboración propia. 
mayor relación de los tuits con la cuenta de la serie de ficción que con el perfil de Netflix Estados Unidos, revelando un uso escaso del hashtag o la mención correspondiente. Sin embargo, el contenido de los tuits analizados nos permite hallar la conexión del showrunner con las estrategias de marketing e imagen de marca características de esta plataforma, ya que dichos profesionales comparten los eventos y el material promocional emitido por Netflix a través de sus perfiles.

Finalmente, se aprecia la posibilidad de comparar los datos obtenidos con los índices de audiencia de las series de televisión para valorar la existencia de una relación directa entre el comportamiento de los showrunners en Twitter y el consumo del producto televisivo. En este caso, la ausencia de información explícita sobre la audiencia de todos sus proyectos por parte de la plataforma de Netflix limitaría la citada propuesta. No obstante, se contempla la ampliación futura de la investigación mediante la aplicación del modelo a una muestra diferente de perfiles de showrunners para contrastar y complementar los resultados obtenidos.

\section{Referencias bibliográficas}

Aguirre-Sacasa, R. [@WriterRas]. (25 de octubre 2018). One more pic to celebrate the \#CAOS drop that's happening tonight at midnight on \#Netflix. Kiernan and Chance, on the [Image attached] [Tweet]. Twitter. http://bit.ly/2rq9g89

Aguirre-Sacasa, R. [@WriterRas]. (5 de abril 2019). Are you seeing doublesdoubles? Then you must be watching Ep. 119 of \#Sabrinanetflix. Will the prophecy be fulfilled?? \#CAOS [Image attached] [Tweet]. Twitter. http://bit.ly/2qC6qgj

Buschow, C., Schneider, B. \& Ueberheide, S. (2014). Tweeting television: Exploring communication activities on Twitter while watching TV. Communications. The European Journal of Communication Research, 39(2), 129-149. https://doi.org/10.1515/commun-2014-0009

Calderón Kellett, G. [@everythingloria]. (8 de febrero de 2019).Next up 5808 Sunset Blvd. free @OneDayAtATime food truck!! Come out \& let us feed you \& give you high [Tweet]. Twitter. http://bit. ly/2DdP0sS 
Carrillo-Bernal, J. (2018). Paradigma Netflix. El entretenimiento de algoritmo. Editorial UOC.

Cascajosa-Virino, C. (2016). El ascenso de los "showrunners": Creación y prestigio crítico en la televisión contemporánea. Index Comunicación, 6(2), 23-40. https://bit.ly/2vKauxw

Cascajosa-Virino, C. (2018). Las series de televisión españolas ante la llegada de los servicios VOD (2015-2017). El Profesional de la Información, 27(6),1303-1312. https://doi.org/10.3145/epi.2018.nov.13

Castro-Mariño, D. (2016). La audiencia social y el mundo narrativo de la ficción: Análisis de los comentarios de los fans y community managers sobre la ficción televisiva y webseries españolas. Palabra Clave, 21(1), 86-110. https://doi.org/10.5294/pacla.2018.21.1.5

Cornelio-Marí, E. M. (2020). Melodrama mexicano en la era de Netflix: algoritmos para la proximidad cultural. Comunicación y Sociedad, e7481, 1-27. https://doi.org/10.32870/cys.v2020.7481

Deller, R. (2011). Twittering on: Audience research and participation using Twitter. Participations. Journal of Audience \& Reception Studies, 8(1), 216-245. http://bit.ly/33d4QPK

DuVernay, A. [@ava]. (22 de mayo de 2019). America was never great. There was always someone at the bottom. Someone being oppressed, enslaved, interned, caged, deported. How did [Video attached] [Tweet]. Twitter. http://bit.ly/37DZlwb

Fernández-Gómez, E. \& Martín-Quevedo, J. (2018). La estrategia de engagement de Netflix España en Twitter. El Profesional de la Información, 27(6), 1292-1302. https://doi.org/10.3145/epi.2018.nov.12

Fernández-Manzano, E. P., Neira, E. \& Clares-Gavilán, J. (2016). Gestión de datos en el negocio audiovisual: Netflix como estudio de caso. El Profesional de la Información, 25(4), 568-576. https://doi. org/10.3145/epi.2016.jul.06

Gallego, F. (2013). Social TV analytics: Nuevas métricas para una nueva forma de ver televisión. Index Comunicación, 3(1), 13-39. http:// bit.ly/2JJ76H4

García-Avilés, J. A. (2012). Roles of audience participation in multiplatform television: From fans and consumers, to collaborators and activists. Participations. Journal of Audience \& Reception Studies, 9(2), 429-447. http://bit.ly/2NcICYR 
Guerrero, E., Diego, P. \& Kimber, D. (2017). Hooked on lit screens. El Profesional de la Información, 26(6), 1108-1117. https://doi. org/10.3145/epi.2017.nov.10

Guo, M. (2019). Social television viewing with second screen platforms: Antecedents and consequences. Media and Communication, 7(1), 139-152. http://dx.doi.org/10.17645/mac.v7i1.1745

Halpern, D., Quintas-Froufe, N. \& Fernández-Medina, F. (2016). Interacciones entre la televisión y su audiencia social: Hacia una conceptualización comunicacional. El Profesional de la Información, 25(3), 367-375. https://doi.org/10.3145/epi.2016.may.06

Harrington, S., Highfield, T. \& Bruns, A. (2013). More than a backchannel: Twitter and Television. Participations. Journal of Audience \& Reception Studies, 10(1), 405-409. http://bit.ly/2JGz46g

Hederson, J. [@Henderson_Joe]. (8 de mayo de 2019). A year ago,we were cancelled. Today, \#LuciferSeason4 is on Netflix!! These episodes are for you, Lucifans. Thanks for fighting [Tweet]. Twitter. http://bit.ly/2DjSIRG

Higueras-Ruiz, M. J., Gómez-Pérez, F. J. \& Alberich-Pascual, J. (2018). Historical review and contemporary characterization of showrunner as professional profile in TV series production: traits, skills, competences, and style. Communication \& Society, 31(1), 91-105. http:// bit.ly/326nPtV

Higueras-Ruiz, M. J., Gómez-Pérez, F. J. \& Alberich-Pascual, J. (2019). Revisión histórica y conceptual de la autoría y sus implicaciones en el medio televisivo: el concepto de autor en las series de televisión contemporáneas estadounidenses. Doxa Comunicación, (28), 7996. https://doi.org/10.31921/doxacom.n28a04

Izquierdo-Castillo, J. (2015). El nuevo negocio mediático liderado por Netflix: Estudio del modelo y proyección en el mercado español. El Profesional de la Información, 24(6), 819-826. https://doi. org/10.3145/epi.2015.nov.14

James, S. E. (2017). Early organization, commercialization and weaponization of social media in the entertainment industry: A case study, Bones, season 6 (2010-2011). Cogent Social Sciences, 3(1), 1-28. https://doi.org/10.1080/23311886.2017.1313927 
Jenner, M. (2014). Is this TVIV? On Netflix, TVIII and bingewatching. New Media \& Society, 18(2), 257-273. https://doi. org/10.1177/1461444814541523

Kemp, S. (2019). Digital 2019: Global Internet Use Accelerates. We are Social. http://bit.ly/2NarTp1

Lacalle-Zalduendo, C. \& Gómez-Morales, B. (2017). La recepción televisiva española en la era multipantalla. Comunicación y Sociedad, (30), 197-216. https://doi.org/10.32870/cys.v0i30.6135

Latorre-Martínez, P., Orive-Serrano, V. \& Íñiguez-Dieste, D. (2018). Medición y análisis de la audiencia social de las televisiones autonómicas en Facebook y Twitter. El Profesional de la Información, 27(5), 1061-1070. https://doi.org/10.3145/epi.2018.sep.10

Lin, J. S. \& Peña, J. (2013). Are you following me? A content analysis of TV networks' brand communication on Twitter. Journal of Interactive Advertising, 12(1), 17-29. https://doi.org/10.1080/15252019 .2011 .10722188

Lotz, A. D. (2016). The paradigmatic evolution of U.S. television and the emergence of internet-distributed television. Icono 14, 14(2), 122-142. https://doi.org/10.7195/ri14.v14i2.993

Marwick, A. E. \& boyd, d. (2010). I tweet honestly, I tweet passionately: Twitter users, context collapse, and the imagined audience. New Media \& Society, 13(1), 114-133. https://doi. org/10.1177/1461444810365313

Matrix, S. (2014). The Netflix effect: Teens, binge watching, and on-demand digital media trends. Jeunesse: Young People, Texts, Cultures, 6(1), 119-138. https://doi.org/10.1353/jeu.2014.0002

McNutt, M. (2018). Social TV fandom and the media industries. Transformative Works and Cultures, 26, 1-7. https://doi.org/10.3983/ twc.2018.1504

Mittell, J. (2015). Complex TV. The poetics of contemporary television storytelling. New York University Press.

Navar-Gill, A. (2017). From strategic retweets to group hangs: Writers' room Twitter accounts and the productive ecosystem of TV social media fans. Television \& New Media, 19(5), 415-430. https://doi. org/10.1177/1527476417728376 
Navar-Gill, A. (2018). The fan/creator alliance: Social media, audience mandates, and the rebalancing of power in studio-showrunner disputes. Media Industries Journal, 5(2), 19-34. http://dx.doi.org/ 10.3998/mij.15031809.0005.202

Newman, M. Z. \& Levine, E. (2012). Legitimating Television: Media Convergence and Cultural Status. Routledge.

Ojer, T. \& Capapé, E. (2013). Netflix: A business model in the distribution of audiovisual content. Journalism and Mass Communication, 3(9), 575-584. http://bit.ly/2pzBi0f

Pearson, R. (2010). Fandom in the digital era. Popular Communication, 8(1), 84-95. https://doi.org/10.1080/15405700903502346

Phalen, P. F. (2018). Writing Hollywood. The work and professional culture of television writers. Routledge.

Quintas-Froufe, N. \& González-Neira, A. (2014). Audiencias activas: Participación de la audiencia social en la televisión. Comunicar, 22(43), 83-90. https://doi.org/10.3916/C43-2014-08

Quintas-Froufe, N. \& González-Neira, A. (2016). Consumo televisivo y su medición en España: Camino hacia las audiencias híbridas. El Profesional de la Información, 25(3), 376-383. https://doi. org/10.3145/epi.2016.may.07

Rincón, O. (2011). Nuevas narrativas televisivas: Relajar, entretener, contar, ciudadanizar, experimentar. Comunicar, 18(36), 43-50. https://doi.org/10.3916/C36-2011-02-04

Scolari, C. A. \& Establés, M. J. (2017). El ministerio transmedia: Expansiones narrativas y culturas participativas. Palabra Clave, 20(4), 1008-1041. https://doi.org/10.5294/pacla.2017.20.4.7

Segado, F., Grandío, M. M. \& Fernández-Gómez, E. (2015). Social media and television: A bibliographic review on the Web of Science. El Profesional de la Información, 24(3), 227-234. https://doi. org/10.3145/epi.2015.may.02

Simons, N. (2014). Audience reception of cross-and transmedia TV drama in the age of convergence. International Journal of Communication, 8, 1120-1139. http://bit.ly/2N8vIe5

Van-Esler, M. (2016). Not yet the post-TV era: Network and MVPD adaptation to emergent distribution technologies. Media and Communication, 4(3), 131-141. http://dx.doi.org/10.17645/mac. v4i3.548 
Wayne, M. L. (2018). Netflix, Amazon, and branded television content in subscription video on-demand portals. Media, Culture \& Society, 40(5), 725-741. https://doi.org/10.1177/0163443717736118

Wright, B. [@bradtravelers]. (12 de diciembre de 2018). \#Travelers drops this Friday Dec 14th! So, if you like season 3, tell the world! Good reviews and ratings on [Tweet]. Twitter. http://bit.ly/2ruyMJ6 